\title{
UPGRADE TO INITIAL BPM ELECTRONICS MODULE AND BEAMLINE COMPONENTS FOR CALIBRATION OF THE LEDA BEAM POSITION MEASUREMENTS*
}

\author{
D. Barr, J. D. Gilpatrick, R. Shurter, Los Alamos National Laboratory.
}

\begin{abstract}
The Low-Energy Demonstration Accelerator (LEDA), designed and built at the Los Alamos National Laboratory, is part of the Accelerator Production of Tritium (APT) program and provides a platform for measuring high-power proton beam-halo formation. Beam Position Monitors (BPMs) are placed along the FODO lattice and the HEBT. The BPM systems employing logratio processor electronics have recently been upgraded for all fifteen BPMs along the accelerator. Two types of calibration are now used. The first corrects for errors within the electronics module and the log-amp transfer function non-conformity. The second is a single-point routine used to correct for cable plant attenuation differences. This paper will also cover the new switching systems used for various system calibration modes as well as various results from LEDA beam runs. New switching algorithms were implemented in order to remove sensitive electronic switches from within the beam tunnel radiation environment. Attention will be paid to the calibration algorithms and switching system interactions, and how well they work in practice.
\end{abstract}

\section{BPM CONFIGURATION AND USE}

The LEDA BPM system provides several services to the commissioning team. First, it provides information that allows the centering of the beam in the HEBT and into the beamstop. Secondly, it allows verification of the quadrupole magnetic field settings by offsetting the beam position from magnetic lattice center [1].

The position measurement systems consist of microstripline BPMs, coaxial cables, analog front-end (AFE) processing systems including Analog Devices AD8307 logarithmic amplifiers, real-time error correction via digital hardware, digital signal processing chips, and control system interfaces. The beamline probes themselves are a four-electrode micro-stripline design. For more details and a photograph see [2]. For details of the AFE and AD8307 log-amplifier see [3,4].

It is possible to use one of several common processor transfer functions for implementing a beam position measurement system. These include difference-over-sum, arctan, log ratio, and normalized power difference functions [5]. The LEDA system uses the log-ratio technique. Advantages of the log-ratio system over other systems include improved sensitivity and linearity. The

* Supported by US DOE, Office of Defense Programs, and

the Office of Nuclear Energy, Science and Technology. main drawback is ripple errors due to the approximation nature of real-world log amplifiers [6]. They use a cascade of nonlinear cells to approximate the logarithmic function in series of piecewise-linear segments. This error is corrected using the calibration technique detailed in a later section of this paper.

It is necessary to understand the individual characteristics of each BPM before they are placed in the beamline. A mapping technique performed in a wellequipped laboratory is necessary to understand the sensitivities needed for the log ratio calculation [2].

\section{SIGNAL PROCESSING AND DATA ACQUISITION}

A VXI module has been developed to interface the analog beamline sensors to the accelerator control system, EPICS (Experimental \& Physics Industrial Control System). The same VXI module can be used for all beam position measurements and contains an on-board calibrator. Two internal TMS320C40 DSP modules control the calibration, data processing, and general operation of the VXI module.

The signal from the upstream ports of the BPMs are run over long-length (approximately 170 feet) rf coaxial cables and fed into the inputs labeled in Figure 1. The BPM AFE processes this raw $350 \mathrm{MHz}$ signal through various stages including the logarithmic amplifier ending up at a 14-bit A/D converter. See [3, 4] for more details. Only twelve bits are used $[4,6]$. This signal is used to address a 4096 location RAM look-up table (RAMLUT). These RAMLUTs are located on the AFEs themselves and are addressed using Field-Programmable Gate Arrays (FPGAs). The RAMLUTs are twelve bits wide, but the values are sign-extended to sixteen bits before being passed to another set of FPGAs downstream labeled DSP FPGA \#1 and \#2. These FPGAs are used to calculate the sum and/or differences of the raw lobe signals as shown in Figure 2. For more details see [6].

\section{STANDARD SYSTEM CALIBRATION}

The ideal response of a logarithmic amplifier can be stated as

$$
V_{\text {out }}=K \cdot \log \left(\frac{v_{\text {in }}}{v_{\text {ref }}}\right)
$$


where $v_{\text {in }}$ is the amplitude of the input signal, $v_{\text {ref }}$ is a reference voltage, and $\mathrm{K}$ is an amplitude constant [6]. Both $v_{\text {ref }}$ and $\mathrm{K}$ are dependent on the particular log amp chosen. Since the log amp is non-ideal, there are small perturbations in its transfer function and distortion effects at the upper and lower ends of its dynamic range. By using digital error-correction the non-ideal performance in the log amps and other system non-linearities can be taken out of the overall transfer function of the system. The system calibration provides a method to determine the error correction values.

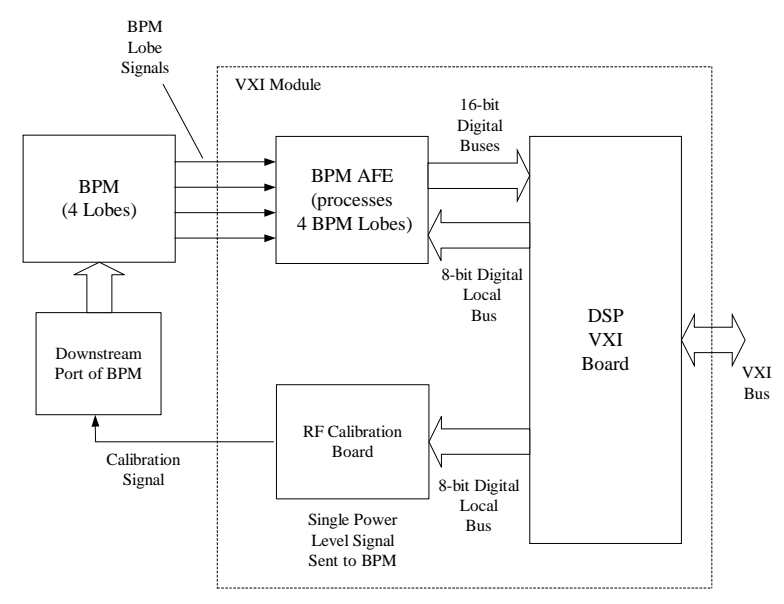

Figure 1: Previously Used Method for Calibration

As stated in the previous section, the error correction takes place in the RAMLUTs. The 12-bit digitized lobe signals from the analog section are used as an address for the 4096-point (12-bit) deep RAMLUT. The 12-bit wide output word is used as the new corrected lobe input into the DSP FPGAs.

The system calibration is a method for placing known values into the downstream ports of the BPM and measuring the generated output signal at the DSP. The RAMLUTs are bypassed for this measurement. This calibration output data is compared to the theoretical values that would be obtained at the DSP with the known input power levels. Since the response of the system transfer function depends so much on the dynamic range of the log amp, the calibration must be done over a large range of power levels. This is accomplished by means of a bank of attenuators on a printed-circuit daughter card. A fixed amount of power is generated at $350 \mathrm{MHz}$ on the calibrator board and then attenuated in 1-db steps over a range of 128 steps. With an input power of $25 \mathrm{dBm}$ (which translates to about $17 \mathrm{dBm}$ due to the cable and splitter), it is possible to calibrate over the full dynamic range of the log amplifiers. Once this data is collected, a linear interpolation routine is used to generate the remaining values in the 4096-deep RAMLUT. This effectively fills in the gaps between the 1-dB steps with a LSB of $0.03 \mathrm{~dB}$. The RAMLUT tables are calculated offline using LabVIEW System Software from National Instruments. The table values are loaded into the
RAMLUTs using the 8-bit local bus shown in Figures 1 and 2. A test routine was written to check how well the RAMLUTs work once loaded. This "test calibration" routine is performed immediately after a successful calibration and works as follows. A range of signal powers is placed into the downstream ports of the BPMs by means of the attenuators on the calibrator board. The values for these signals are measured at the DSPs as with the calibration routine. The main difference is that now the RAMLUTs are used to process the data. This measured data is subtracted from the theoretical values that would be obtained at the DSP with the known input power levels. These values are now plotted versus attenuation, thus obtaining an estimate of system performance over the full dynamic range.

\section{IMPROVED SYSTEM CALIBRATION}

It is possible to calibrate the full-dynamic range of the electronics using a scheme as outlined in Figure 2. This would apply the full attenuator range test locally on the VXI module. A single-power level signal would be sent along the calibration path shown in Figure 1. A splitter is used to split the signal to the four BPM lobes. The primary hardware benefit of this scheme is the removal of a series of switches placed in the beamline for switching the calibration signal through the BPM probes. Experience has shown the unreliability of such devices and the impracticalities of maintenance and possible replacement. Since only one power level is needed, it is possible to use a lower level than the maximum required for the full-dynamic range test. The full-dynamic test through the beamline required switches since a high enough power level could not be generated with a splitter and accompanying attenuator. The attenuator is needed to give a high enough isolation between the legs. In the present case, a Wilkinson four-way splitter is used. It has an isolation of $30 \mathrm{~dB}$ between the legs. With an added 20 $\mathrm{dB}$ pad for each leg, the total isolation is an acceptable 70 $\mathrm{dB}$. An added benefit is that since a lower power level is needed for the calibration through the probes, a cheaper and more reliable RF source can be placed on the RF calibration board.

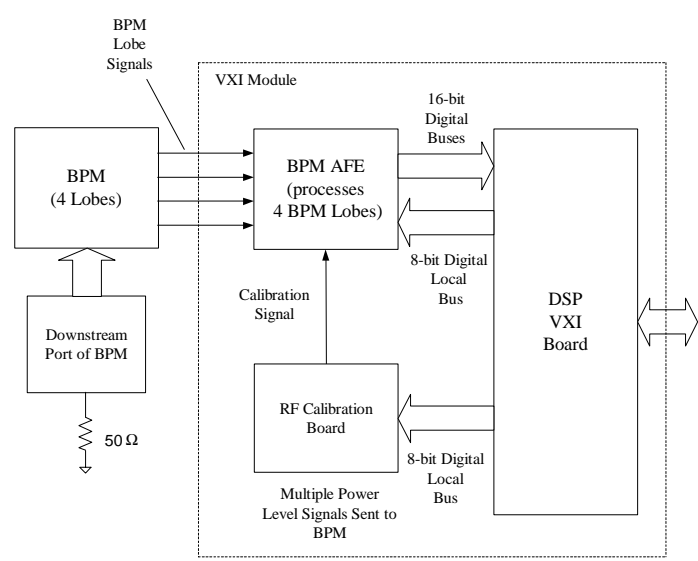

Figure 2: Improved Calibration Scheme 


\section{ATTENUATOR CHARACTERIZATION}

In order to better understand the calibration procedure it is necessary to understand the imperfections in the attenuators used to generate the calibration signals themselves. Each of the fifteen calibrators was characterized in the lab using an HP 8753 network analyzer. Any imperfections were noted and stored in a table. The theoretical values used in the calibration process now take into account the individual characteristics of the attenuators loaded from these tables.

\section{POSITION UNCERTAINTY}

An error assessment was performed of all known absolute errors, such as mechanical displacement errors of the BPM mapping, BPM installation, cable mismatch errors, and electronics errors. The resulting root of the sum of the squares of the known absolute errors is summarized in Table 1.

Table 1: RMS Error Analysis for BPMs

\begin{tabular}{|l|l|}
\hline BPM Mapping Error & 119 microns \\
\hline $\begin{array}{l}\text { Mechanical } \\
\text { Installation Error }\end{array}$ & 23 microns \\
\hline Electronics & 25 microns \\
\hline Cables Mismatch & 100 microns \\
\hline \hline \\
$\begin{array}{l}\text { Total } \\
\text { Uncertainty }\end{array}$ \\
\hline
\end{tabular}

\section{ANALYZED RELATIVE ERRORS}

The graph in Figure 3 was created from data derived from a series of calibration test runs taken under typical running conditions from the lattice BPMs. These data were taken from BPM 29 that was one of the BPMs showing the smallest level of error. See [1] for an example using one of the lattice BPMs with the highest level of relative error. From the Figure 3 it is seen that both the horizontal and vertical maximum errors were below $0.025 \mathrm{~mm}$ over the 1 to $100 \mathrm{ma}$ operating range of the accelerator. It is clear that the calibration procedure is calibrating the BPMs to less than two LSBs of the 0.025 $\mathrm{mm}$ maximum position error over the accelerator operating current range using the lattice BPMs. The bunched beam current maximum error was within $0.2 \%$ of the distribution's nominal value.

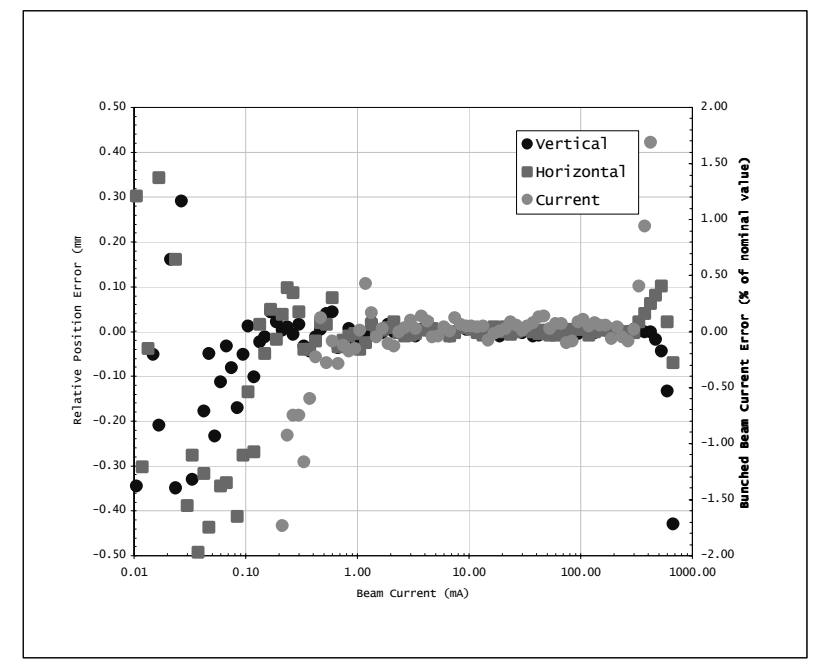

Figure 3: Analyzed Relative Error for a Typical BPM

\section{SUMMARY}

The system worked extremely well and very reliably. The addition of the attenuator characterization files decreased system error. The calibration test routine showed that the total absolute errors for horizontal and vertical position are well within specified tolerance [1].

\section{REFERENCES}

[1] J.D. Gilpatrick, et al., "Experience with the Low Energy Demonstration Accelerator (LEDA) Halo Experiment Beam Instrumentation," published at this conference.

[2] J.D. Gilpatrick, et al, LEDA \& APT Beam Position Measurement System: Design and Initial Tests, Proceedings of the 1998 Linac Conference.

[3] R.B. Shurter, et al., "Analog Front-End Electronics for Beam Position Measurement on the Beam Halo Measurement," published at this conference.

[4] R.B. Shurter, R. B., et. al, "BPM Analog Front-End Electronics Based on the AD8307 Log Amplifier," Proceedings of the 2000 Beam Instrumentation Workshop, Boston, MA.

[5] J.D. Gilpatrick, "Comparison of Beam-Position Transfer Functions Using Circular Beam-Position Monitors," Proceedings of the 1997 Particle Accelerator Conference, Vancouver, B.C., Canada.

[6] D. Barr, et al., "LEDA Beam Diagnostics Instrumentation: Beam Position Monitors," Proceedings of the 2000 Beam Instrumentation Workshop, Boston, MA. 